\title{
Electrophysiological evidence of the time course of attentional bias in non-patients reporting symptoms of depression with and without co-occurring anxiety
}

\section{Sarah M. Sass ${ }^{1}{ }^{*}$,Wendy Heller ${ }^{2}$, Joscelyn E. Fisher ${ }^{3}$, Rebecca L. Silton ${ }^{4}$, Jennifer L. Stewart ${ }^{5}$, Laura D. Crocker ${ }^{2}$, J. Christopher Edgar ${ }^{6}$, Katherine J. Mimnaugh ${ }^{2}$ and Gregory A. Miller ${ }^{2,7,8}$}

${ }^{1}$ Department of Psychology, University of Texas at Tyler, Tyler, TX, USA

${ }^{2}$ Department of Psychology, University of Illinois at Urbana-Champaign, Champaign, IL, USA

${ }^{3}$ Department of Psychiatry, Uniformed Services University of the Health Sciences, Bethesda, MD, USA

${ }^{4}$ Department of Psychology, Loyola University Chicago, Chicago, IL, USA

${ }^{5}$ Department of Psychiatry, University of California at San Diego, San Diego, CA, USA

${ }^{6}$ Department of Radiology, University of Pennsylvania, Philadelphia, PA, USA

${ }^{7}$ Department of Psychology, University of California at Los Angeles, Los Angeles, CA, USA

${ }^{8}$ Department of Psychiatry and Biobehavioral Sciences, University of California at Los Angeles, Los Angeles, CA, USA

\section{Edited by:}

Daniela M. Pfabigan, University of

Vienna, Austria

\section{Reviewed by:}

Ulrich Ansorge, University of Vienna,

Austria

Susan Jennifer Thomas, University of

Wollongong, Australia

\section{${ }^{*}$ Correspondence:}

Sarah M. Sass, Department of

Psychology, University of Texas at

Tyler, 3900 University Boulevard,

HPR223, Tyler, TX 75799, USA

e-mail:ssass@uttyler.edu
Anxiety is characterized by attentional biases to threat, but findings are inconsistent for depression. To address this inconsistency, the present study systematically assessed the role of co-occurring anxiety in attentional bias in depression. In addition, the role of emotional valence, arousal, and gender was explored. Ninety-two non-patients completed the Penn State Worry Questionnaire (Meyer etal., 1990; Molina and Borkovec, 1994) and portions of the Mood and Anxiety Symptom Questionnaire (Watson etal., 1995a,b). Individuals reporting high levels of depression and low levels of anxiety (depression only), high levels of depression and anxiety (combined), or low levels of both (control) completed an emotion-word Stroop task during event-related brain potential recording. Pleasant and unpleasant words were matched on emotional arousal level. An attentional bias was not evident in the depression-only group. Women in the combined group had larger N200 amplitude for pleasant than unpleasant stimuli, and the combined group as a whole had larger right-lateralized P300 amplitude for pleasant than unpleasant stimuli, consistent with an early and later attentional bias that is specific to unpleasant valence in the combined group. Men in the control group had larger N200 amplitude for pleasant than unpleasant stimuli, consistent with an early attentional bias that is specific to pleasant valence. The present study indicates that the nature and time course of attention prompted by emotional valence and not arousal differentiates depression with and without anxiety, with some evidence of gender moderating early effects. Overall, results suggest that co-occurring anxiety is more important than previously acknowledged in demonstrating evidence of attentional biases in depression.

Keywords: attentional bias, anxiety, depression, emotion, event-related brain potentials

\section{INTRODUCTION}

An impressive body of research has demonstrated that depression and anxiety are characterized by cognitive biases, including attentional bias or preferential attentional processing of unpleasant or threatening information. Attentional bias has been argued to contribute to the etiology and maintenance of anxiety and depression (e.g., Beck, 1976; Beck et al., 2005; Bar-Haim et al., 2007; Levin et al., 2007; Gotlib and Joormann, 2010). Whereas anxiety has been consistently associated with attentional biases to threatening or emotionally arousing stimuli (Williams et al., 1996; McNally, 1998; Becker etal., 2001; Fox etal., 2002), evidence of attentional biases in depression has been mixed (Mogg and Bradley, 2005; Gotlib and Joormann, 2010), with some studies finding preferential processing of unpleasant stimuli (Gotlib and Cane, 1987; Bradley et al., 1997), others insufficient attention to pleasant stimuli (Gotlib et al., 1988; McCabe and Gotlib, 1995; Gilboa and Gotlib, 1997; McCabe et al., 2000), and others a lack of differentiation between pleasant and unpleasant stimuli (e.g., McCabe and Gotlib, 1995; Deldin et al., 2001).

Along with inconsistent evidence regarding the nature of attentional biases in depression, evidence regarding the time course of bias has also been inconsistent. Understanding the time course of attentional processing is critical in elucidating the degree to which early registration and vigilance, relying primarily on early sensory processing involving brain areas such as visual cortex and amygdala, or later, more elaborative attentional and cognitive-control mechanisms, relying primarily on cortical and prefrontal regions (for reviews, see Bishop, 2007; Gotlib and Joormann, 2010), are involved in abnormal attention to emotional information. Some evidence exists for an early attentional bias in favor of unpleasant 
information in depression, especially from electrophysiological studies (e.g., Williams et al., 2007; Jaworska et al., 2010). Evidence of a later bias in favor of unpleasant information in depression also exists (e.g., Gotlib and Joormann, 2010), supporting the hypothesis that later attentional processes (such as difficulty disengaging from or inhibiting unpleasant information) are involved in attentional biases in depression (e.g., Leyman et al., 2007; Joormann and D'Avanzato, 2010).

Depression and anxiety are frequently co-occurring and share considerable conceptual and measurement overlap (Clark and Watson, 1991; Heller et al., 1998; Keller et al., 2000; Nitschke et al., 2001; Watson, 2009). Surprisingly few studies account for anxiety in attentional bias investigations of depression (Bar-Haim etal., 2007), despite the potential for co-occurring anxiety to affect the nature or timing of bias in depression. A large literature documents evidence of both early (e.g., Williams et al., 1996; Bar-Haim et al., 2007; Eysenck et al., 2007; Li et al., 2007; Sass etal., 2010) and later (e.g., Fox et al., 2002; Li et al., 2007) attentional biases in anxiety, with evidence of attention being captured quickly by threatening stimuli (e.g., Williams et al., 1996), difficulty disengaging from threatening stimuli once attended (e.g., Fox et al., 2002), initial engagement with threatening stimuli followed by avoidance (e.g., Mogg etal., 2004), and preferential engagement with both pleasant and threatening compared to neutral information (e.g., Martin etal., 1991; Sass et al., 2010). In samples with depression and co-occurring anxiety, evidence of attentional bias is sometimes found (e.g., Rossignol et al., 2008; Markela-Larenc et al., 2011) and sometimes not (Bradley et al., 1995).

Co-occurring anxiety can also affect patterns of brain activity in regions implementing attentional control (e.g., Heller, 1990, 1993; Heller et al., 1995; Heller and Nitschke, 1997; Keller et al., 2000). Although anxiety is a broad construct that is often treated monolithically, anxious apprehension (worry) and anxious arousal (panic or sympathetic arousal), are accompanied by distinct patterns of abnormal activity in brain regions implementing attentional control (e.g., Heller et al., 1997; Heller and Nitschke, 1998; Engels et al., 2007, 2010) and are associated with a dissociable time course of attentional bias to emotionally arousing stimuli (Sass et al., 2010). In order to examine the neural mechanisms involved in the time course of attentional disruption in depression with and without co-occurring anxiety, it is important to investigate these dimensions of anxiety in conjunction with depression.

Different patterns of attentional biases depending on depression and anxiety co-occurrence could have substantial implications for treatment. Unique cognitive characteristics of depression with and without anxiety (and potential targets for intervention) could be associated with different neural mechanisms. Co-occurring anxiety can affect patterns of brain activity that are related to attentional processing (e.g., Heller, 1990, 1993; Heller et al., 1995; Heller and Nitschke, 1997; Keller et al., 2000; Engels et al., 2010). Resting EEG, functional magnetic resonance imaging (fMRI), and event-related brain potential (ERP) studies provide evidence of lateralization patterns in depression with less right than left activity over parieto-temporal regions (e.g., Deldin et al., 2000; Engels et al., 2010; Stewart et al., 2011). These posterior brain regions are associated with vigilance and autonomic arousal, and less activity in these areas in depressive states is presumably due to less arousal characterized by symptoms such as anhedonia (e.g., Heller and Nitschke, 1998; Engels et al., 2010). For example, ERP studies demonstrate evidence of reduced right parietal N200 amplitude (Deldin et al., 2000) and P300 amplitude (Sumich et al., 2006) in depressed individuals. Conversely, depression with co-occurring anxious apprehension has been associated with greater right inferior occipital cortex fMRI activity and co-occurring anxious arousal with greater right inferior temporal gyrus fMRI activity in response to unpleasant vs. neutral information in the context of an emotion-word Stroop task (Engels et al., 2010). Examining lateralized neural mechanisms reflecting the time course of processing of emotional stimuli may provide critical insights in understanding biased processing of emotional stimuli in depression with and without co-occurring anxiety.

In addition to co-occurring anxiety, emotional valence and emotional arousal are important to systematically investigate in attentional biases in depression. Pleasant stimuli are inconsistently included in attentional bias studies and when they are included, are not consistently matched to unpleasant stimuli on emotional arousal level (e.g., see Mogg and Bradley, 2005, Table 1; Williams etal., 1996). It is possible that a general emotional arousal confound contributes to variance in findings. That is, it may be the high emotional arousal value of unpleasant stimuli and not unpleasant valence per se that drives attentional biases in depression. In order to assess this issue, the present study matched unpleasant and pleasant stimuli on emotional arousal level.

Gender is also important to investigate systematically in attentional bias research. Women are estimated to suffer from depression and anxiety twice as often as men (Weissman et al., 1996; Nolen-Hoeksema, 2001; Craske, 2003). Several studies indicate that gender moderates emotional information processing in depressed (e.g., Wright etal., 2009) and anxious (e.g., Sass et al., 2010) participants. For example, depressed women took longer to categorize negative faces than did control women, whereas depressed men performed no differently than control men (Wright et al., 2009). Failing to include gender may further contribute to inconsistency regarding the nature and timing of

Table 1 | Means and standard deviations for questionnaire scores used to form groups.

\begin{tabular}{llll}
\hline & \multicolumn{3}{c}{ Group Questionnaire Scores } \\
\cline { 2 - 4 } & PSWO & MASQ-AA & MASQ-AD \\
\hline Group & & & \\
Depression-only & $36(9.4)$ & $22(2.5)$ & $25(2.8)$ \\
Combined & $71(5.4)$ & $71(5.4)$ & $27(3.9)$ \\
Control & $38(8.6)$ & $20(2.2)$ & $13(2.4)$ \\
\hline
\end{tabular}

PSWQ refers to the Penn State Worry Questionnaire. MASQ-AA and MASQ-AD refer to the anxious arousal and anhedonic depression subscales of the Mood and Symptom Questionnaire, respectively. 
attentional biases in depression and may unnecessarily limit understanding of how these biases contribute to and maintain depression. Gender also moderates processing of emotional stimuli in control participants, with women sometimes showing evidence of preferential processing of unpleasant stimuli (e.g., Lang etal., 1998), and men tending to show the opposite pattern, prioritizing pleasant information (Lang et al., 1998; Bradley and Lang, 2007). A more comprehensive understanding of the nature and time course of the processing of emotional stimuli in control participants may provide useful information regarding the higher prevalence rates of depression and anxiety in women.

In examining attention to emotional stimuli in depression and anxiety, many studies have used an emotion-word variant of the Stroop task. Distracter word content is unpleasant ("assault"), neutral ("cabinet"), or pleasant ("festive"), and participants are asked to ignore the content or meaning of the word while responding to the color of the word. A recent meta-analysis indicated that clinically depressed individuals show slower color naming in the emotion-word Stroop task for unpleasant than for neutral words, consistent with biased processing of unpleasant information (Epp et al., 2012). Similarly, in anxiety, a large literature demonstrates that color naming is slowed in anxious participants when the distracter word is unpleasant or threatening, with larger effects in individuals diagnosed with anxiety disorders and smaller or inconsistent effects in individuals with self-reported trait or state anxiety (e.g., Williams et al., 1996; Koven et al., 2003; Bar-Haim et al., 2007). Reaction time (RT) alone may not be a precise indicator of attentional bias given that delayed RT can be interpreted as avoidance instead of heightened attention toward negative stimuli (e.g., De Raedt and Koster, 2010). In contrast, ERP methodology offers high temporal resolution that can differentiate early sensory from later more elaborative processing prior to response selection and execution. In general, early sensory processing occurs prior to 300 ms (e.g., Luck et al., 2000), and later conflict detection processes occur beginning approximately 300-600 ms (e.g., Donchin and Coles, 1988; Coles et al., 2000; West, 2003).

The present study focused on P100 and posterior visual N200 amplitude as indices of earlier, more automatic stimulus processing, and P300 (sometimes called P3b, late positive potential (LPP), or late positive complex (LPC) as an index of later, more elaborative stimulus processing. P100 amplitude peaks approximately $100 \mathrm{~ms}$ after stimulus onset and grows larger as more extrastriate cortex resources are devoted to processing stimuli (Luck etal., 2000). P100 was larger for sad than for joyful facial expressions (Jaworska et al., 2010) and smaller for positive words in depressed than in control participants (Dai and Feng, 2011). In anxious participants in the emotion-word Stroop task, P100 was larger for unpleasant than neutral stimuli (e.g., Li et al., 2007) and for unpleasant and pleasant than neutral stimuli (Sass et al., 2010).

Posterior visual N200 (what is sometimes called N100) immediately follows P100 over occipito-parietal sensors (e.g., Allison et al., 2002; Ruz and Nobre, 2008; Sass et al., 2010), but peaks later (approximately $200 \mathrm{~ms}$ ) than classical N100 elicited in visual attention tasks (e.g., Gonzalez et al., 1994; Anllo-Vento and Hillyard, 1996), especially those using short intertrial intervals (ITIs).
N200 likely originates in extrastriate cortex and is maximal over bilateral occipital-posterior regions [sometimes called early posterior negativity (EPN); e.g., Weber et al., 2009]. In depression, smaller N200 for happy than sad faces (e.g., Deldin et al., 2000) or no modulation of $\mathrm{N} 200$ amplitude in response to emotional stimuli (e.g., Kayser et al., 2000) has been found. In anxiety, larger N200 amplitude has been associated with processing emotionally arousing than neutral stimuli in the context of an emotion-word Stroop task (Sass et al., 2010). Taken together, P100 and N200 amplitude results indicate stronger evidence for an early bias in anxiety but mixed evidence for an early bias in depression, mirroring the behavioral literature. The posterior N200 component in the present study can be distinguished from a fronto-central N200 component that is thought to be associated with effortful processing (such as inhibition and conflict monitoring; e.g., Donkers and van Boxtel, 2004), and which typically peaks later in time (between 200 and 500 ms; e.g., Thomas et al., 2007; Enriquez-Geppert et al., 2010).

P300 amplitude is associated with context updating and event categorization processes (e.g., Donchin and Coles, 1988) as well as increased resource engagement (e.g., Yee and Miller, 1994). P300 amplitude is often modulated by emotional arousal, with larger amplitude for emotionally arousing than neutral stimuli interpreted as reflecting more attentional resources devoted to processing these stimuli (e.g., Schupp et al., 2004; Fischler and Bradley, 2006; Thomas et al., 2007; Li et al., 2007; Franken et al., 2009; Sass et al., 2010). In anxiety, P300 amplitude has been larger for unpleasant than neutral words (Li et al., 2007) and for emotionally arousing (pleasant and unpleasant) than neutral words (no difference between anxious and control participants, Sass et al., 2010) in the context of an emotion-word Stroop task. In comorbid anxiety and depression, no P300 effects were found in a visual oddball task including happy, sad, and neutral faces (Rossignol et al., 2008). Thus, inconsistent P300 amplitude evidence exists for a later attentional resource allocation bias for unpleasant or emotionally arousing stimuli in both depression and anxiety.

In order to address questions concerning the role of emotional valence, emotional arousal, co-occurring anxiety, and gender on the nature and timing of attentional biases in depression, the present study examined ERPs in three groups of participants: depression only (scored high on an 8-item Mood and Anxiety Symptom Questionnaire (MASQ) anhedonic depression measure and low on anxiety measures), combined (scored high on Penn State Worry Questionnaire (PSWQ) and MASQ measures of anxiety and high on an 8-item MASQ anhedonic depression measure), or control (scored low on anxiety and depression measures. The control group was included in order to investigate whether patterns of preferential attentional processing of unpleasant or emotionally arousing stimuli were specific to the depression only or combined groups. Pleasant and unpleasant stimuli were matched on emotional arousal level.

Critical differences in the nature and timing of attention to emotion were explored in the three groups. (1) It was unclear whether early effects would be present in the depression only or combined group, given inconsistency in the literature of early effects in depression and a general lack of consideration of 
co-occurring anxiety. If attentional bias is relatively automatic and specific to unpleasant stimuli in depression with and without anxiety, then P100 and/or posterior visual N200 amplitude should be larger for unpleasant than pleasant words. Alternatively, if initial bias is more broadly associated with emotional arousal, then P100 and/or posterior visual N200 amplitude should be larger for both unpleasant and pleasant than neutral words. (2) Later effects were predicted to occur in depression with and without co-occurring anxiety, given a literature documenting later effects in both depression and anxiety. If later, more strategic processing is specific to unpleasant stimuli in depression with and without co-occurring anxiety, then P300 amplitude should be larger for unpleasant than pleasant words. Alternatively, if later, more strategic processing is more broadly associated with emotional arousal, then P300 amplitude should be larger for both unpleasant and pleasant than neutral words. (3) If unpleasant or emotionally arousing words are attended followed by avoidance, than P100 and/or posterior visual N200 amplitude should be larger and P300 amplitude smaller for unpleasant or emotionally arousing stimuli in depression with and without co-occurring anxiety. (4) Gender may moderate early or later attentional processing and was included as an exploratory variable in present analyses. (5) P100, posterior visual N200, and P300 effects may be more pronounced over right posterior regions in the combined group and less pronounced in the depressed group, consistent with previous research regarding regional EEG, ERP, and $\mathrm{fMRI}$ patterns in depression with and without co-occurring anxiety (e.g., Heller and Nitschke, 1998; Keller et al., 2000; Sumich et al., 2006; Engels et al., 2010).

\section{MATERIALS AND METHODS}

Much of the methods section, including stimuli and experimental design, EEG recording procedure, and data reduction and analysis procedures overlap with Sass et al. (2010) and to some extent with Fisher et al. (2010) and Stewart et al. (2010). Method details are included here in slightly modified (not verbatim) form from Sass et al. (2010).

All participants provided informed consent, and all procedures were approved for ethical considerations by the University of Illinois Institutional Review Board. A total of 4,457 college undergraduates were screened for the study. Participants were 92 (49 female) paid volunteers (mean age $=19.0, \mathrm{SD}=1.9$ ) recruited via group questionnaire screening sessions ${ }^{1,2}$. Participants were

\footnotetext{
${ }^{1}$ Ninety-six participants qualified for the present study. Of these, two were disqualified due to missing RT data, and two were disqualified due to having one or more ERP component scores (P100, N200, or P300 amplitude) $>3$ SD from the mean.

${ }^{2}$ Participants from the present study overlapped with three previous ERP investigations. Fifty-nine participants in the present sample were included in Stewart et al. (2010). Stewart et al. (2010) investigated attentional biases as a function of approach and withdrawal anger styles and did not focus on depression with and without cooccurring anxiety. That study also focused on different ERP components than the present study, including N100, P200, N200, and N400 amplitude at fronto-central and central sites, not investigated in the present study. The only overlapping ERP component investigated in Stewart et al. (2010) and the present study is P300 amplitude, with different sensors used. Forty-two participants were shared with Fisher et al. (2010), who investigated emotional information processing as a function of perceived emotional intelligence and focused on different ERP components including P100 and P300 amplitude (defined using different temporal scoring windows and different sensors than the present study), and P200 and slow wave (neither used in the present study). Finally, 32 controls from the present study were used
}

82\% European American and were classified as high anhedonic depression ( $n=24 ; 11$ female), combined ( $n=27 ; 19$ female), or control ( $n=41 ; 19$ female) on the basis of responses on the PSWQ and MASQ. Compared to the total sample screened for the study, the anhedonic depression group scored at or above the 80th percentile on an eight-item depressed-mood subscale (Nitschke et al., 2001) of the MASQ Anhedonic Depression scale, shown to predict diagnostic category membership (Bredemeier et al., 2010). The anhedonic depression group also scored at or below the 50th percentile on the PSWQ and on the MASQ Anxious Arousal scale. The combined group scored at or above the 80th percentile on all three scales. The control group scored at or below the 50th percentile on all three scales. Table 1 presents the means and standard deviations of the groups for the questionnaire measures.

The Structured Clinical Interview for Axis I Disorders, NonPatient Edition (First et al., 1997), was administered to all participants to assess to what degree selecting participants based on the questionnaire measures yielded significant Axis I disorders. Participants were not selected based on DSM diagnosis, because DSM-defined anxiety and depression disorders include (to varying degrees) anxious apprehension, anxious arousal, and anhedonic depression. Selecting participants based on DSM category would likely result in missed sensitivity in uncovering brain mechanisms (e.g., see Engels et al., 2007, 2010; Herrington et al., 2010) distinctly associated with attentional processing as a function of the transdiagnostic dimensions of anhedonic depression and anhedonic depression co-occurring with anxious arousal and anxious apprehension.

Lifetime DSM-IV-TR (American Psychiatric Association, 2000) diagnoses were determined by a trained clinical psychology doctoral student interviewer and reviewed by a consensus team consisting of a second trained clinical psychology doctoral student interviewer and a clinical faculty supervisor (Gregory A. Miller). Although participants were not selected based on DSMIV-TR depression or anxiety disorder diagnosis, approximately $25 \%$ of the individuals in the depression only and $59 \%$ of the combined group met criteria for a lifetime history of major depressive disorder (MDD) and/or an anxiety disorder. Specifically, of the 24 individuals in the depressed group, six met full criteria for a lifetime history of MDD. Of the 27 individuals in the combined group, 11 had a lifetime history of MDD (three were in a current major depressive episode) and 13 had a lifetime history of an anxiety disorder (anxiety disorder not otherwise specified, generalized anxiety disorder, obsessive compulsive disorder, posttraumatic stress disorder, social phobia). Control participants did not meet criteria for any lifetime DSM-IV-TR disorder. Therefore, the questionnaire measures used to screen individuals for the present combined and depressed groups provided a substantial number of participants meeting criteria for DSM-IV-TR diagnoses of MDD and/or an anxiety disorder.

The groups did not differ in age. All participants were determined to be right-handed by the Edinburgh Handedness Inventory (Oldfield, 1971), had self-reported normal color vision, and

in Sass et al. (2010), which focused on ERP evidence of the timing of attention to emotional information in pure anxious apprehension vs. pure anxious arousal. 
were native speakers of English. Participants were informed of the procedures of the study and given a laboratory tour. Exclusion criteria included DSM-IV-TR alcohol or drug abuse or dependence within the past 3 months, experience with electroshock therapy, multiple sclerosis, epilepsy, current pregnancy, claustrophobia, moderate to severe head injury, loss of consciousness for $10 \mathrm{~min}$ or more, and contraindications for MRI participation (including metal present in the body).

\section{STIMULI AND EXPERIMENTAL DESIGN}

STIM software (James Long Company, Caroga Lake, NY, USA) controlled word presentation and button-press response recording. Neutral blocks were interleaved between blocks of pleasant and unpleasant emotion words. Two hundred fifty-six words were delivered to participants in 16 blocks (four pleasant, eight neutral, four unpleasant) of 16 trials. A word was presented in the center of the computer screen for $1500 \mathrm{~ms}$ at the beginning of each trial, followed by a fixation cross for 275 to $725 \mathrm{~ms}$ (onsetto-onset ITI $2000+/-225 \mathrm{~ms}$ ). Each trial consisted of a single word presented in one of four colors (red, yellow, green, blue) on a black background. Each color appeared equally often with each word type (pleasant, neutral, unpleasant). Participants completed an emotion-word Stroop task in both EEG and fMRI sessions that were counterbalanced to precede each other equally often. The present report is based on the EEG data. Participants were randomly assigned one of eight possible orders. In half of the presentation orders, the first and third blocks were neutral words, and the second and fourth blocks were pleasant and unpleasant, with valence order counterbalanced across participants. In the remaining half of the presentation orders the first and third blocks were either pleasant or unpleasant emotion words and the second and fourth blocks were neutral words. These eight presentation orders were designed to avoid order effects by ensuring that the neutral and emotional words preceded each other equally often. A given word was presented only once per session to control stimulus familiarity. Each color appeared four times within a block and no more than two trials featuring the same color appeared in a row. A brief rest period occurred after every fourth block. In addition to 16 word blocks, four fixation blocks were presented: one at the beginning, one at the end, and two in the middle of the experiment. Specifically, a bright fixation cross was presented for $1500 \mathrm{~ms}$ instead of a word, followed by a dimmer fixation cross that always followed word stimuli.

Sixty-four pleasant, 64 unpleasant, and two sets of 64 neutral words were carefully selected from the Affective Norms for English Words set (ANEW; Bradley and Lang, 1999) on the basis of norms for valence, arousal, and frequency of usage in the English language (Bradley and Lang, 1999). Pleasant and unpleasant words were chosen to be high in arousal (arousal mean $=6.53$ for pleasant, 6.56 for unpleasant, and 3.81 for neutral stimuli). Words ranged from three to eight letters in length and were presented in capital letters using Tahoma 72-point font. The visual display was $1.35 \mathrm{~m}$ from the participant's eyes for a vertical span of $1.5^{\circ}$ and a horizontal span of $2.5-9.3^{\circ}$. The average luminance values of the words presented in red, yellow, green, or blue were 15, 72, 45, and 20 lux, respectively. Verbatim instructions were read by experimenters to ensure consistency. Each participant performed
32 practice trials before the actual task began. There were four buttons on the response box, with each button corresponding to a color. The left middle and index fingers were used to indicate red and green, respectively. The right middle and index fingers were used to indicate yellow and blue, respectively. All participants understood task instructions and the mapping between colors and buttons after the practice trials were completed.

\section{EEG RECORDINGS}

Participants were seated in a quiet room that was connected via intercom to an adjacent room where the experimenter controlled EEG data collection and stimulus presentation. A custom Falk Minow 64-channel cap with equidistantly spaced $\mathrm{Ag} / \mathrm{AgCl}$ electrodes was used to record EEG. The left mastoid was the online reference for all EEG and electrooculogram (EOG) sites. Vertical and horizontal EOG was recorded with electrodes placed above and below each eye and near the outer canthus of each eye for off-line eye-movement artifact correction of EEG. Electrode impedances were below 20 Kohms. Data were digitized at $250 \mathrm{~Hz}$, and half-power amplifier bandpass was $0.1-100 \mathrm{~Hz}$. A Zebris ELPOS digitizer recorded electrode positions (Zebris Medizintechnik, Tübingen, Germany).

\section{DATA REDUCTION}

Muscle, movement, and other artifacts were removed manually. Eye blinks were corrected using Brain Electrical Source Analysis (BESA 5.1.8) software (Berg and Scherg, 1994). If a channel was off-scale for more than $10 \%$ of trials, all trials for that channel for a given subject were removed from analyses. All channels for epochs in which a single channel was off-scale were discarded. Artifact correction did not differentially affect the number of remaining pleasant, neutral, and unpleasant trials, Emotion $F(2,89)=1.45$, $p=0.237^{3}$, and did not differ by group or gender. Only correct trials were averaged for the pleasant, neutral, and unpleasant conditions. The electrode configuration was transformed using spherical spline interpolation to BESA's standard 81-channel montage (Perrin et al., 1989), reflecting the 10-10 system. An average reference (the mean voltage over the 81 standard virtual scalp electrodes) was computed for each time point, and data were exported from BESA. Each channel was baseline-adjusted in custom Matlab software by subtracting the average amplitude for the $200 \mathrm{~ms}$ before stimulus onset.

Three ERP components were scored: P100 (88-128 ms), N200 (160-240 ms), and P300 (448-580 ms). A 101-weight, 0.1-20 Hz digital filter was used for P100 and N200, and a 101-weight, 0.1$8 \mathrm{~Hz}$ digital filter was used for P300 (Cook and Miller, 1992; Nitschke et al., 1998; Edgar et al., 2005). A combination peak/area measure was used to avoid spurious peaks driving amplitude measures. Voltage $48 \mathrm{~ms}$ around the peak was averaged for the early (P100, N200) components, and voltage $96 \mathrm{~ms}$ around the peak was averaged for the late (P300) component. Latency associated with each peak was also recorded.

Sites for P100 and N200 were chosen based on examination of current source density (CSD) estimates across conditions and

${ }^{3}$ Because the number of neutral trials was double the number of each emotion condition, this analysis involved dividing the number of neutral trials by two and then comparing the number in each condition after artifact removal. 

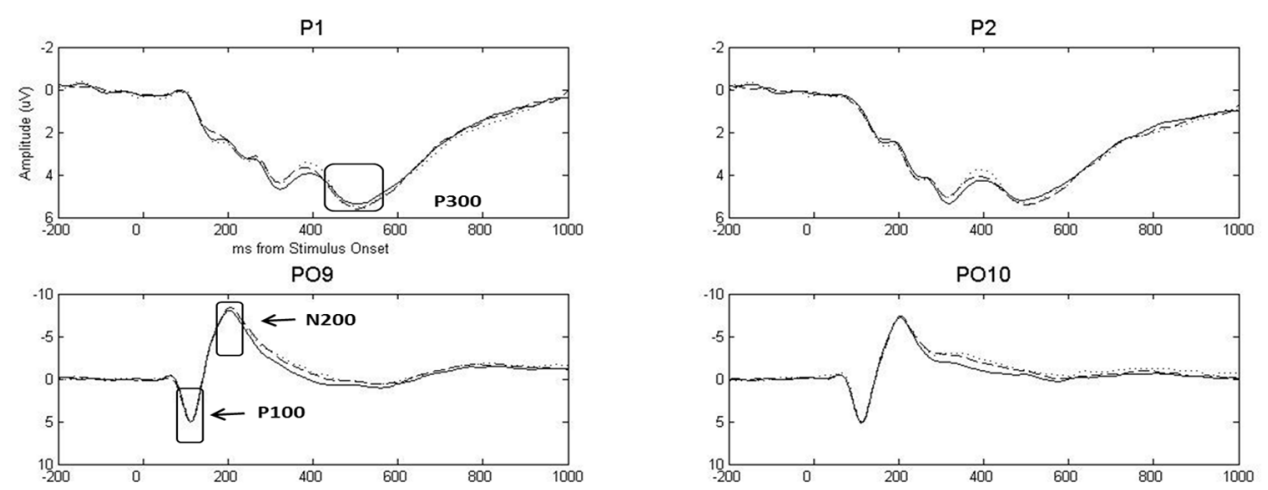

FIGURE 1 | Grand-average event-related potential waveforms for representative posterior sensors, highlighting P100, N200, and P300. Dotted, solid, and dashed lines represent pleasant, neutral, and unpleasant stimuli, respectively. Stimulus onset was at time $=0 \mathrm{~ms}$.

groups. CSD estimates were used as an estimate of the contribution of the immediately underlying cortical surface to the recorded electrode signal (Nunez et al., 1999). Voltage associated with amplitude values at sites where CSD activity was maximal for P100 (P7, P8, PO7, PO8, O1, O2) and N200 (P7, P8, P9, P10, PO7, PO8, PO9, PO10) were averaged together by hemisphere for these bilaterally distributed components. Voltage associated with amplitude values at sites for P300 (P1, P2, P3, P4, CP1, CP2, CP3, CP4) were averaged by hemisphere. Sites for $\mathrm{P} 300$ were chosen based on previous emotion-word Stroop studies (e.g., van Hooff et al., 2008; Sass et al., 2010) and inspection of the grand-average waveforms where effects were maximal.

\section{RESULTS}

\section{BEHAVIORAL PERFORMANCE}

Pleasant, neutral, and unpleasant RT was analyzed for correct trial responses between 350 and $1400 \mathrm{~ms}(M=671 \mathrm{~ms}$, $\mathrm{SD}=106 \mathrm{~ms}) .4 .5 \%$ of RT data were lost due to the RT criterion of $<350 \mathrm{~ms}$ or $>1400 \mathrm{~ms}$. Performance accuracy was high (mean number of errors $=4.0, \mathrm{SD}=4.1$, of 256 trials). Participants were excluded from EEG analyses if they were excluded from RT analyses, and from RT analyses if they were excluded from EEG analyses. A Group (depression only, combined, control) x Gender (female, male) x Emotion (pleasant, neutral, unpleasant) multivariate analysis of variance (MANOVA) was conducted. Levels of the emotion factor were ordered pleasant, neutral, and unpleasant in order to take advantage of a priori orthogonal linear (valence: comparing pleasant with unpleasant) and quadratic (arousal: comparing pleasant and unpleasant with neutral) univariate trends on the emotion factor. All tests were 2-tailed using an alpha level of 0.05 and $p$-values reflect the Huynh-Feldt correction for sphericity where appropriate. No main effects or interactions were significant for RT.

\section{EARLY EMOTION-WORD PROCESSING}

A Group (depression only, combined, control) x Gender (female, male) $x$ Emotion (pleasant, neutral, unpleasant) $\mathrm{x}$ Hemisphere (left, right) MANOVA including linear and quadratic trends (described above) was conducted separately for P100 and N200 (see Figure 1 for grand-average waveforms for representative channels). Reported $p$-values reflect the Huynh-Feldt correction for sphericity where appropriate.

\section{P100 amplitude}

P100 was larger over right than left hemisphere, $F(1,86)=19.67$, $p<0.001$, and a Group main effect, $F(2,86)=3.93, p=0.023$, was qualified by a Group $x$ Hemisphere interaction, $F(2,86)=3.09$, $p=0.050$. Separate Group ANOVAs were done for each hemisphere. The Group effect was significant over left, $F(2,89)=3.34$, $p=0.040$, and right, $F(2,89)=4.29, p=0.017$, hemispheres, dissected with orthogonal Group contrasts for each hemisphere. The first contrast compared the combined with the depressed group, and the second contrast pooled depressed groups and compared them with controls. P100 amplitude was smaller over left hemisphere in the depressed than combined group, $p=0.015$, and the combined group did not differ from controls (see Figure 2). P100 amplitude was smaller over right hemisphere in both depressed

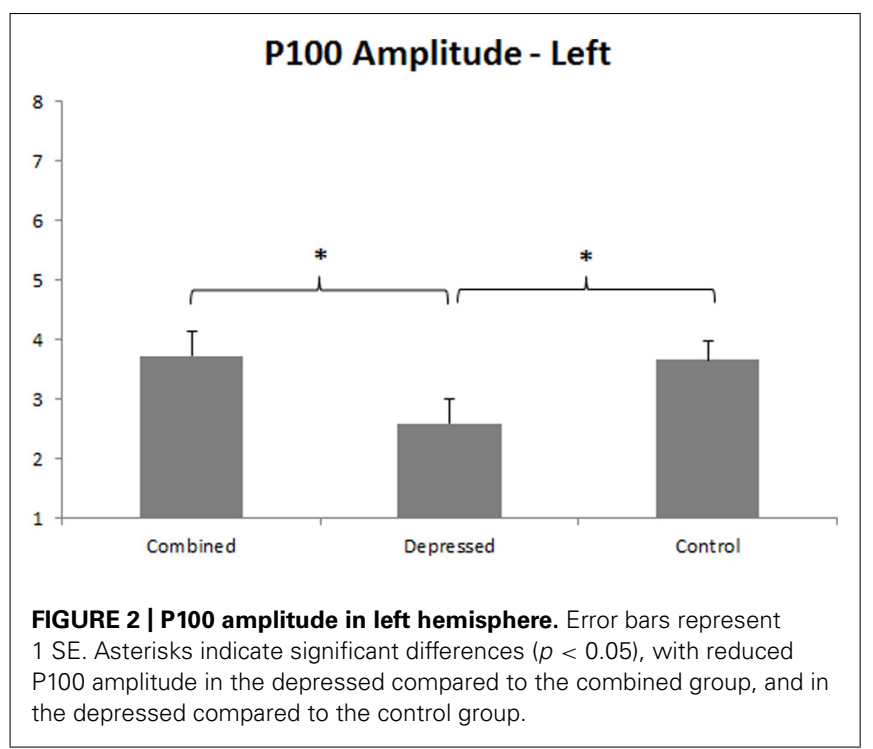




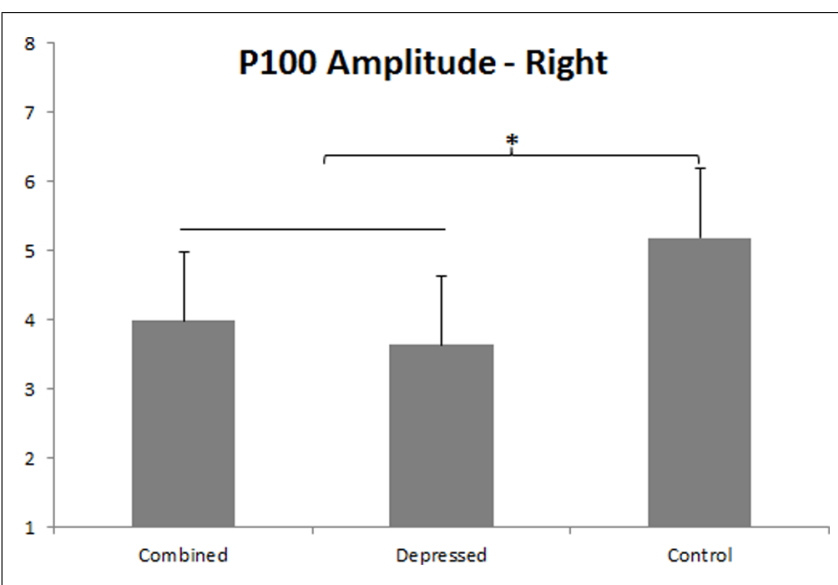

FIGURE 3 | P100 amplitude effect in right hemisphere. Error bars represent 1 SE. The asterisk indicates ( $p<0.05$ ), that both depressed groups showed reduced P100 amplitude compared to the control group.

groups compared to controls, $p=0.005$, and the depressed and combined group did not differ from one another (see Figure 3).

\section{N200 amplitude}

N200 amplitude was larger over left than right hemisphere, $F(1,86)=7.59, \quad p=0.007$. A main effect of Emotion, $F(2,86)=3.52, p=0.032$, was evaluated with linear and quadratic contrasts. N200 amplitude was larger for emotionally arousing than neutral words, $F(1,86)=11.43, p=0.010$. In addition, a Gender $x$ Emotion effect, $F(2,86)=3.40, p=0.036$, was qualified by a Group $\mathrm{x}$ Gender $\mathrm{x}$ Emotion trend, $F(4,86)=2.32$, $p=0.059$. This latter interaction was dissected with Gender $\mathrm{x}$ Emotion ANOVA analyses for each group, following hypotheses
1, 3, and 4. A Gender $x$ Emotion interaction was present in the combined, $F(2,25)=3.83, p=0.028$, and control, $F(2,39)=4.51$, $p=0.013$, but not depressed group. This interaction was dissected with separate Emotion ANOVAs for each gender within the combined and control groups using linear and quadratic contrasts. In the combined group, N200 amplitude was larger for unpleasant than pleasant words in women but not men, linear $F(1,18)=5.00$, $p=0.038$. In the control group, N200 amplitude was larger for pleasant than unpleasant words in men but not women, linear $F(1,21)=9.65, p=0.005$ (see Figure 4).

\section{LATER EMOTION-WORD PROCESSING \\ P300 amplitude}

A Group x Gender x Emotion x Hemisphere MANOVA including linear and quadratic trends (described above) was conducted for P300 amplitude. An Emotion effect, $F(2,86)=3.95, p=0.021$, was followed up with linear and quadratic contrasts. As expected, P300 amplitude was larger for pleasant and unpleasant than neutral words, quadratic Emotion $F(1,86)=7.22, p=0.009$. A Gender $\mathrm{x}$ Hemisphere interaction, $F(1,86)=8.84, p=0.004$, was investigated with separate Hemisphere ANOVAs for each gender. Only men had larger P300 amplitude over the right than left hemisphere, $F(1,42)=4.72, p=0.036$. A Group x Hemisphere effect, $F(1,86)=3.51, p=0.034$, was qualified by a Group $\mathrm{x}$ Emotion $\mathrm{x}$ Hemisphere interaction, $F(4,86)=2.44, p=0.049$, dissected with separate Emotion $\mathrm{x}$ Hemisphere ANOVAs for each group, following hypotheses 2, 3, and 5. A linear Emotion $\mathrm{x}$ Hemisphere interaction, $F(2,26)=3.32, p=0.044$, emerged in the combined group only. This interaction was dissected with separate Emotion ANOVAs for each hemisphere within the combined group using linear and quadratic contrasts on the emotion factor. P300 amplitude was larger for unpleasant than pleasant stimuli over the right

\section{N200 Amplitude}

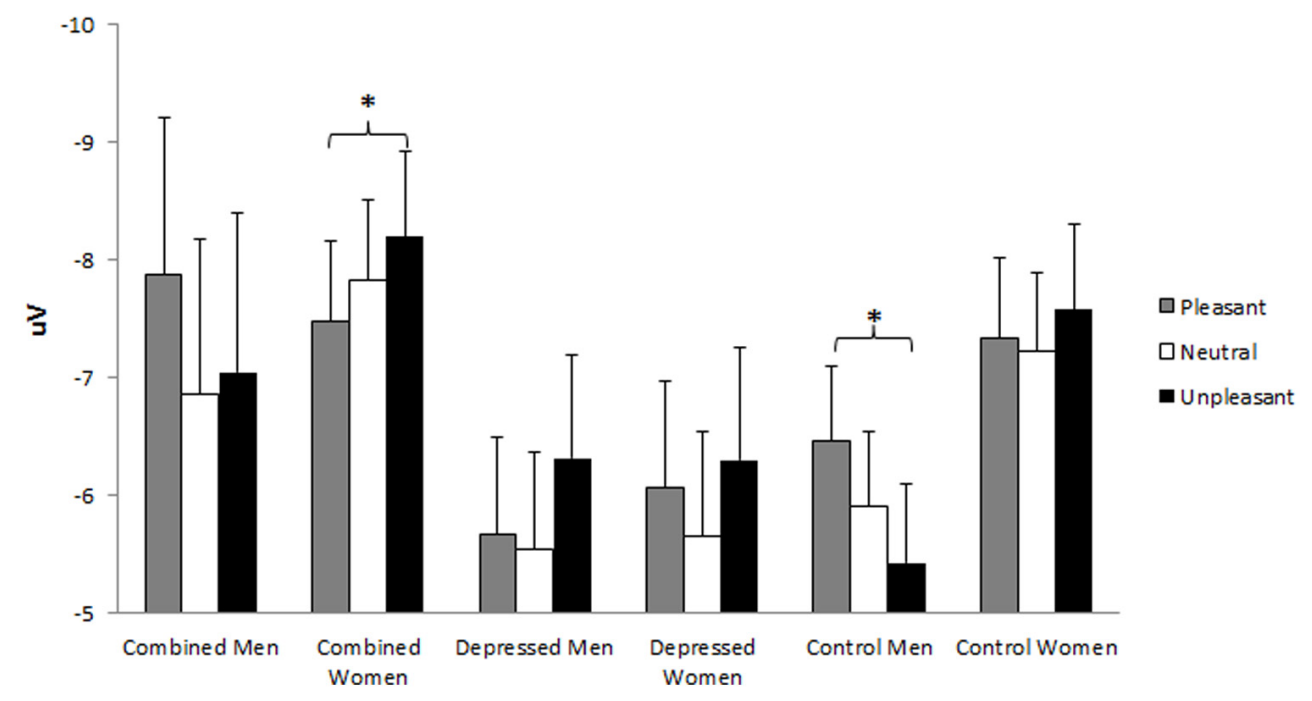

FIGURE 4 | N200 amplitude. Error bars represent 1 SE. N200 amplitude valence effect in combined women and control men. The asterisks represent significant differences with $p<0.05$ in combined women (unpleasant > pleasant) and control men (pleasant $>$ unpleasant). 


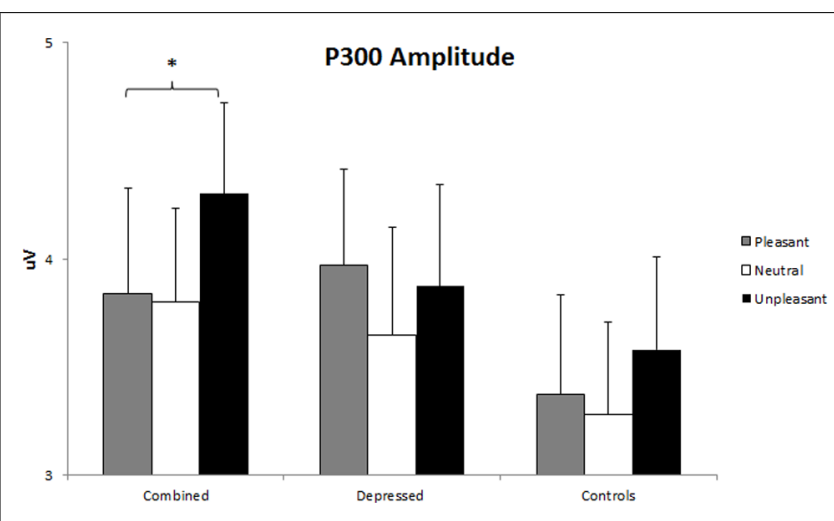

FIGURE 5 | P300 amplitude over right hemisphere. Error bars represent 1 SE. P300 amplitude valence effect (unpleasant > pleasant) over right hemisphere sensors (P1, P3, CP1, CP3) in the combined group. The asterisk represents $p<0.05$ in the combined group (unpleasant $>$ pleasant).

but not left hemisphere, linear $F(1,25)=4.35, p=0.047$ (see Figure 5).

\section{DISCUSSION}

Previous literature provides inconsistent evidence regarding the nature and timing of attentional biases in depression, and unexamined co-occurring anxiety may contribute to this inconsistency. The present study investigated the role of co-occurring anxiety and whether early, relatively automatic, or later, less automatic phenomena manifested in the ERP evidence would support attentional bias in favor of unpleasant or emotionally arousing stimuli in depression. Gender was included as an exploratory variable.

Evidence of early biased processing of unpleasant or emotionally arousing stimuli was absent in the depression-only group. The combined group, however, showed evidence of both an early and a later attentional bias in favor of unpleasant information. Posterior N200 amplitude was larger in women with both depression and anxiety symptoms for unpleasant than for pleasant stimuli, presumably reflecting relatively automatic processing of unpleasant words, consistent with other emotion-word Stroop studies finding modulation of an EPN for emotional compared to neutral stimuli (Franken et al., 2009; Sass et al., 2010). That an early effect modulated by emotion was not evident in the depressiononly group suggests that high levels of anxiety are necessary to elicit evidence of attentional bias in depression, consistent with findings of Markela-Larenc et al. (2011).

The combined depression and anxiety group also showed evidence of biased processing later in the trial (larger P300 amplitude for unpleasant than pleasant over right hemisphere), reflecting biased processing of unpleasant information at a later, more elaborative stage. This finding is consistent with emotion-word Stroop studies showing P300 amplitude modulation by emotion (e.g., Li et al., 2007; Franken et al., 2009; Sass et al., 2010). This finding is also consistent with studies finding greater right-lateralized posterior brain activity in depression co-occurring with anxiety (e.g., Engels et al., 2010).

The later preferential attention to unpleasant information seen in the combined group was not observed in the depression-only group. The present depression sample was unusual in that participants were selected only if they scored high on a measure of anhedonic depression and low (in the control group range) on two measures of anxiety, allowing the relatively pure influence of anhedonic depression to be examined. Given present evidence of attentional bias effects in the combined but not depressed group, results indicate that attentional bias effects sometimes found in depressed samples may be due to co-occurring anxiety.

The depression-only group produced smaller P100 amplitude over left hemisphere than the combined group, consistent with EEG studies showing less left than right hemisphere activity in depressed compared to anxious individuals. These findings are also consistent with fMRI results revealing reduced left dorsolateral prefrontal cortical activity in depression when co-occurring anxiety is taken into account (Engels et al., 2010; Herrington et al., 2010).

In addition, both depressed groups showed reduced P100 amplitude over right hemisphere compared to controls that was not specific to emotional stimuli. This result suggests that depressed groups' detection of visual stimuli is generally dampened at this early time point ( $\sim 100 \mathrm{~ms})$, despite early ( $\sim 200 \mathrm{~ms})$, and later ( $~ 500 \mathrm{~ms})$ ERP effects showing differential detection and processing of unpleasant stimuli in the combined group. This effect is in contrast to early P100 amplitude effects modulated by anxiety in previous emotion-word Stroop studies (Li et al., 2007; Sass et al., 2010), suggesting that high levels of anxiety and lower levels of depression are necessary to elicit early $(\sim 100 \mathrm{~ms})$ emotion effects. P100 amplitude was not modulated by emotion in the present study, consistent with a number of previous studies failing to find early emotion effects in depression (e.g., Rossignol et al., 2008; Gotlib and Joormann, 2010).

There are several caveats and limitations associated with the present study. First, RT evidence of attentional bias was not obtained in the present study. RT is the end-stage of a number of different brain processes, and a lack of RT effects despite ERP effects in the present sample indicates biased processing at stages prior to response execution. This pattern of effects is consistent with and informs studies finding small or no RT effect sizes using the emotion-word Stroop task in samples with subclinical depression (for review, see Epp et al., 2012) and anxiety (Koven et al., 2003). Second, a larger sample and equal gender distribution would be better suited to examining gender differences in emotional information processing that may exist in the depression-only and combined groups. Third, because the Group x Gender x Emotion N200 amplitude effect was at trend level, this result should be interpreted tentatively pending replication. Fourth, in averaging across conditions for ERP analyses, pleasant and unpleasant trials were averaged separately before pooling them for comparison to neutral using quadratic contrasts. It is possible that the lower number of trials contributing to the pleasant and unpleasant ERP averages ( $n=64$ for each emotion condition) would contribute to higher amplitude scores than for the neutral averages $(n=128)$, due to the possibility of more noise in the averages with fewer trials. This issue is only relevant to comparisons of emotionally arousing with neutral stimuli and not to comparisons of pleasant with unpleasant stimuli (thus not affecting the main findings in the present paper, of greater N200 and P300 
amplitude for unpleasant than pleasant stimuli in the combined group). Given that signal-to-noise reduction is a function of a ratio of the square root of the number of trials comprising an average, and 64 is a reasonable number of trials to begin with, the difference in the number of trials contributing to the emotionally arousing and neutral averages should not have much differential impact on noise reduction. Furthermore, present emotional arousal main effects for posterior visual N200 and P300 amplitude are consistent with previous research employing an equal number of pleasant, unpleasant, and neutral trials (e.g., Franken et al., 2009), suggesting that the emotional arousal effects found in the present study are not a function of differing trial numbers. Finally, the use of a block design in the present study is helpful in eliciting more sustained emotion effects as might occur in everyday emotional contexts. A block design may not be optimal in distinguishing early, more automatic processing from later, more strategic processing, as topdown expectancy effects may influence early processing (e.g., see van Hooff et al., 2008, for a similar discussion).

Present results can inform interventions for depression with and without co-occurring anxiety. Computerized attentiontraining programs have been successful in modifying attentional bias and reducing symptoms of depression and anxiety (for metaanalyses see Hakamata et al., 2010; Hallion and Ruscio, 2011). In a study targeting mild to moderate depression with mild levels of co-occurring anxiety, participants in an attention-training condition showed a greater reduction in depressive symptoms than did participants receiving a control intervention (Wells and Beevers, 2010). Another study found small improvements in symptom severity after computerized training among students showing mild depression symptoms, but symptoms worsened in those with moderate to severe depression (Baert et al., 2010). In this latter study, co-occurring anxiety was in the mild to moderate range, leaving open the question of whether individuals without cooccurring anxiety would show similar effects. Future research should build on these initial studies, targeting both earlier and later attentional biases and systematically examining the role of co-occurring anxiety.

The present study indicates that the nature and time course of attention prompted by emotional stimuli differentiates depression with and without combined anxiety, and both depressed groups from controls. In the absence of bias effects in the depression-only group, the combined group showed evidence of both an early and a later attentional bias in favor of unpleasant information. Cooccurring anxiety therefore appears to be an important factor in inconsistent results in previous studies regarding attentional biases in depression. Present findings support previous recommendations for careful experimental control of co-occurring anxiety and for including gender and hemisphere when investigating behavioral and brain correlates of attentional biases in depression. Systematic examination of these issues can yield insights into cognition-emotion phenomena in depression that may improve understanding of etiology and treatment, providing valuable directions for future research.

\section{ACKNOWLEDGMENTS}

This research was supported by the National Institute of Mental Health (P50 MH079485, R01 MH61358, T32 MH19554), the
National Institute on Drug Abuse (R21 DA14111), and the University of Illinois Beckman Institute, Department of Psychology, and Intercampus Research Initiative in Biotechnology. The authors thank Chris Murdock and David Towers for their contributions to this project.

\section{REFERENCES}

Allison, T., Puce, A., and McCarthy, G. (2002). Category-sensitive excitatory and inhibitory processes in human extrastriate cortex. J. Neurophysiol. 88, 2864-2868. doi: 10.1152/jn.00202.2002

American Psychiatric Association. (2000). Diagnostic and Statistical Manual of Mental Disorders, 4th Edn, text revision. Washington: American Psychiatric Association.

Anllo-Vento, L., and Hillyard, S. A. (1996). Selective attention to the color and direction of moving stimuli: electrophysiological correlates of hierarchical feature selection. Percept. Psychophys. 58, 191-206. doi: 10.3758/BF03211875

Baert, S., De Raedt, R., Schacht, R., and Koster, E. H. W. (2010). Attentional bias training in depression: therapeutic effects depend on depression severity. J. Behav. Ther. Exp. Psychiatry 41, 265-274. doi: 10.1016/j.jbtep.2010. 02.004

Bar-Haim, Y., Lamy, D., Pergamin, L., Bakermans-Kranenburg, M. J., and van IJzendoorn, M. H. (2007). Threat-related attentional bias in anxious and nonanxious individuals: a meta-analytic study. Psychol. Bull. 133, 1-24. doi: 10.1037/0033-2909.133.1.1

Beck, A. T. (1976). Cognitive Therapy and the Emotional Disorders. New York: International Universities Press.

Beck, A. T., Emery, G., and Greenberg, R. L. (2005). Anxiety Disorders and Phobias: A Cognitive Perspective. New York: Basic Books.

Becker, E. S., Rinck, M., Margraf, J., and Roth, W. T. (2001). The emotional Stroop effect in anxiety disorders: general emotionality or disorder specificity? J. Anxiety Disord. 15, 147-159. doi: 10.1016/S0887-6185(01) 00055-X

Berg, P., and Scherg, M. (1994). A multiple source approach to the correction of eye artifacts. Electroencephalogr. Clin. Neurophysiol. 90, 229-241. doi: 10.1016/00134694(94)90094-9

Bishop, S. J. (2007). Neurocognitive mechanisms of anxiety: an integrative account. Trends Cogn. Sci. 11, 307-316. doi: 10.1016/j.tics.2007.05.008

Bradley, B. P., Mogg, K., and Lee, S. C. (1997). Attentional biases for negative information in induced and naturally occurring dysphoria. Behav. Res. Ther. 35, 911-927. doi: 10.1016/S0005-7967(97)00053-3

Bradley, B. P., Mogg, K., Millar, N., and White, J. (1995). Selective processing of negative information: effects of clinical anxiety, concurrent depression, and awareness. J. Abnorm. Psychol. 104, 532-536. doi: 10.1037/0021-843X. 104.3.532

Bradley, M. M., and Lang, P. J. (1999). Affective Norms for English Words (ANEW): Stimuli, Instruction Manual, and Affective Ratings. Gainesville: The Center for Research in Psychophysiology, University of Florida.

Bradley, M. M., and Lang, P. J. (2007). “Emotion and motivation," in Handbook of Psychophysiology, eds J. T. Cacioppo, L. G. Tassinary, and G. Berntson (New York: Cambridge University Press), 581-607. doi: 10.1017/CBO9780511546396.025

Bredemeier, K., Spielberg, J. M., Silton, R. L., Berenbaum, H., Heller, W., and Miller, G. A. (2010). Screening for depressive disorders using the Mood and Anxiety Symptoms Questionnaire Anhedonic Depression Scale: a receiveroperating characteristic analysis. Psychol. Assess. 22, 702-710. doi: 10.1037/ a0019915

Clark, L. A., and Watson, D. (1991). Tripartite model of anxiety and depression: psychometric evidence and taxonomic implications. J. Abnorm. Psychol. 100, 316-336. doi: 10.1037/0021-843X.100.3.316

Coles, M. G. H., Gratton, G., and Fabiani, M. (2000). "Event related brain potentials," in Principles of Psychophysiology: Physical, Social, and Inferential Elements, eds J. Cacioppo and L. Tassinary (New York: Cambridge University Press), 413-455.

Cook, E. W. III, and Miller, G. A. (1992). Digital filtering: background and tutorial for psychophysiologists. Psychophysiology 29, 350-367. doi: 10.1111/j.14698986.1992.tb01709.x

Craske, M. G. (2003). Origins of Phobias and Anxiety Disorders: Why More Women than Men? Oxford: Elsevier Ltd. 
Dai, Q., and Feng, Z. (2011). Deficient interference inhibition for negative stimuli in depression: an event-related potential study. Clin. Neurophysiol. 122, 52-61. doi: 10. 1016/j.clinph.2010.05.025

Deldin, P. J., Keller, J., Gergen, J. A., and Miller, G. A. (2000). Right posterior face processing anomaly in depression. J. Abnorm. Psychol. 109, 116-121. doi: 10.1037/0021-843X.109.1.116

Deldin, P. J., Keller, J., Gergen, J. A., and Miller, G. A. (2001). Cognitive bias and emotion in neuropsychological models of depression. Cogn. Emot. 15, 787-802. doi: 10.1080/02699930143000248

De Raedt, R., and Koster, E. H. W. (2010). Understanding vulnerability for depression from a cognitive neuroscience perspective: a reappraisal of attentional factors and a new conceptual framework. Cogn. Affect. Behav. Neurosci. 10, 50-70. doi: 10.3758/CABN.10.1.50

Donchin, E., and Coles, M. G. H. (1988). Is the P300 component a manifestation of context updating? Behav. Brain Sci. 11, 357-427. doi $10.1017 /$ S0140525X00058027

Donkers, F. C., and van Boxtel, G. J. (2004). The N200 in go/no-go tasks reflects conflict monitoring not response inhibition. Brain Cogn. 56, 165-176. doi: 10.1016/j.bandc.2004.04.005

Edgar, J. C., Stewart, J. L., and Miller, G. A. (2005). "Digital filtering in EEG/ERP research," in Event-Related Potentials: A Handbook, ed. T. C. Handy (Cambridge: MIT Press), 85-113.

Engels, A. S., Heller, W., Mohanty, A., Herrington, J. D., Banich, M. T., Webb, A. G., et al. (2007). Specificity of regional brain activity in anxiety types during emotion processing. Psychophysiology 44, 352-363. doi: 10.1111/j.1469-8986.2007.00518.x

Engels, A. S., Heller, W., Spielberg, J. M., Warren, S. L., Sutton, B. P., Banich, M. T., et al. (2010). Co-occurring anxiety influences patterns of brain activity in depression. Cogn. Affect. Behav. Neurosci. 10, 141-156. doi: 10.3758/CABN. 10.1.141

Enriquez-Geppert, S., Konrad, C., Pnatev, C., and Huster, R. J. (2010). Conflict and inhibition differentially affect the N200/P300 complex in a combined go/nogo and stop-signal task. Neuroimage 41, 877-887. doi: 10.1016/j.neuroimage.2010. 02.043

Epp, A. M., Dobson, K. S., Dozois, D. J. A., and Frewen, P. A. (2012). A systematic meta-analysis of the Stroop task in depression. Clin. Psychol. Rev. 32, 316-328. doi: 10.1016/j.cpr.2012.02.005

Eysenck, M. W., Derakshan, N., Santos, R., and Calvo, M. G. (2007). Anxiety and cognitive performance: attentional control theory. Emotion 7, 336-353. doi: 10.1037/1528-3542.7.2.336

First, M. B., Spitzer, R. L., Gibbon, M., and Williams, J. B. W. (1997). Structured Clinical Interview for DSM-IV Axis I Disorders: Non-Patient Edition (SCIDI/NP, Version 2.0, 4/97 revision). New York: Biometrics Research, New York State Psychiatric Institute.

Fischler, I. S., and Bradley, M. M. (2006). Event-related potential studies of language and emotion: words, phrases and task effects. Prog. Brain Res. 156, 185-204. doi: 10.1016/S0079-6123(06)56009-1

Fisher, J. E., Sass, S. M., Heller, W., Silton, R. L., Edgar, J. C., Stewart, J. L., et al. (2010). Time course of processing emotional stimuli as a function of perceived emotional intelligence, anxiety, and depression. Emotion 10, 486-497. doi: 10.1037/a0018691

Fox, E., Russo, R., and Dutton, K. (2002). Attentional bias for threat: evidence for delayed disengagement from emotional faces. Cogn. Emot. 16, 355-379. doi: 10.1080/02699930143000527

Franken, I. H., Gootjes, L., and van Strien, J. W. (2009). Automatic processing of emotional words during an emotional Stroop task. Neuroreport 20, 776-781. doi: 10.1097/WNR.0b013e32832b02fe

Gilboa, E., and Gotlib, I. H. (1997). Cognitive biases and affect persistence in previously dysphoric and never-dysphoric individuals. Cogn. Emot. 11, 517-538. doi: 10.1080/026999397379881a

Gonzalez, C. M. G., Clark, V. P., Fan, S., Luck, S. J., and Hillyard, S. A. (1994). Sources of attention-sensitive visual event-related potentials. Brain Topogr. 7, 41-51. doi: 10.1007/BF01184836

Gotlib, I. H., and Cane, D. B. (1987). Construct accessibility and clinical depression: a longitudinal investigation. J. Abnorm. Psychol. 96, 199-204. doi: 10.1037/0021843X.96.3.199

Gotlib, I. H., and Joormann, J. (2010). Cognition and depression: current status and future directions. Annu. Rev. Clin. Psychol. 6, 285-312. doi: 10.1146/annurev.clinpsy.121208.131305
Gotlib, I. H., McLachlan, A. L., and Katz, A. N. (1988). Biases in visual attention in depressed and non-depressed individuals. Cogn. Emot. 2, 185-200. doi: 10.1080/02699938808410923

Hakamata, Y., Lissek, S., Bar-Haim, Y., Britton, J. C., Fox, N. A., Leibenluft, E., et al. (2010). Attention bias modification treatment: a meta-analysis toward the establishment of novel treatment for anxiety. Biol. Psychiatry 68, 982-990. doi: 10.1016/j.biopsych.2010.07.021

Hallion, L. S., and Ruscio, A. M. (2011). A meta-analysis of the effect of cognitive bias modification on anxiety and depression. Psychol. Bull. 137, 940-958. doi: $10.1037 / \mathrm{a} 0024355$

Heller, W. (1990). "The neuropsychology of emotion: developmental patterns and implications for psychopathology," in Psychological and Biological Approaches to Emotion, eds N. Stein, B. L. Leventhal, and T. Trabasso (Hillsdale: Erlbaum), 167-211.

Heller, W. (1993). Neuropsychological mechanisms of individual differences in emotion, personality, and arousal. Neuropsychology 7, 476-489. doi: 10.1037/0894-4105.7.4.476

Heller, W., Etienne, M. A., and Miller, G. A. (1995). Patterns of perceptual asymmetry in depression and anxiety: implications for neuropsychological models of emotion and psychopathology. J. Abnorm. Psychol. 104, 327-333. doi: 10.1037/0021-843X.104.2.327

Heller, W., and Nitschke, J. B. (1997). Regional brain activity in emotion: a framework for understanding cognition in depression. Cogn. Emot. 11, 638-661. doi: $10.1080 / 026999397379845 \mathrm{a}$

Heller, W., and Nitschke, J. B. (1998). The puzzle of regional brain activity in depression and anxiety: the importance of subtypes and comorbidity. Cogn. Emot. 12, 421-447. doi: 10.1080/026999398379664

Heller, W., Nitschke, J. B., Etienne, M. A., and Miller, G. A. (1997). Patterns of regional brain activity differentiate types of anxiety. J. Abnorm. Psychol. 106, 376-385. doi: 10.1037/0021-843X.106.3.376

Heller, W., Nitschke, J. B., and Miller, G. A. (1998). Lateralization in emotion and emotional disorders. Curr. Dir. Psychol. Sci. 7, 26-32. doi: 10.1111/14678721.ep11521823

Herrington, J. D., Heller, W., Mohanty, A., Engels, A. S., Banich, M. T., Webb, A. G., et al. (2010). Localization of asymmetric brain function in emotion and depression. Psychophysiology 47, 442-454. doi: 10.1111/j.1469-8986.2009.00958.x

Jaworska, N., Thompson, A., Shah, D., Fisher, D., Ilivitsky, V., and Knott, V. (2010). Electrocortical effects of acute tryptophan depletion on emotive facial processing in depression-prone individuals. Eur. Neuropsychopharmacol. 20, 473-486. doi: 10.1016/j.euroneuro.2010.03.002

Joormann, J., and D'Avanzato, C. (2010). Emotion regulation in depression: examining the role of cognitive processes. Cogn. Emot. 24, 913-939. doi: $10.1080 / 02699931003784939$

Kayser, J., Bruder, G. E., Tenke, C. E., Stewart, J. E., and Quitkin, F. M. (2000) Event- related potentials (ERPs) to hemifield presentations of emotional stimuli: differences between depressed patients and healthy adults in P3 amplitude and asymmetry. Int. J. Psychophysiol. 36, 211-236. doi: 10.1016/S0167-8760 (00)00078-7

Keller, J., Nitschke, J. B., Bhargava, T., Deldin, P. J., Gergen, J. A., Miller, G. A., et al. (2000). Neuropsychological differentiation of depression and anxiety. J. Abnorm. Psychol. 109, 3-10. doi: 10.1037/0021-843X.109.1.3

Koven, N. S., Heller, W., Banich, M. T., and Miller, G. A. (2003). Relationships of distinct affective dimensions to performance on an emotional Stroop task. Cogn. Ther. Res. 27, 671-680. doi: 10.1023/A:1026303828675

Lang, P. J., Bradley, M. M., Fitzsimmons, J. R., Cuthbert, B. N., Scott, J. D., Moulder, B., et al. (1998). Emotional arousal and activation of the visual cortex: an fMRI analysis. Psychophysiology 35, 199-210. doi: 10.1111/1469-8986.3520199

Levin, R. L., Heller, W., Mohanty, A., Herrington, J. D., and Miller, G. A. (2007). Cognitive deficits in depression and functional specificity of regional brain activity. Cogn. Ther. Res. 31, 211-233. doi: 10.1007/s10608-007-9128-z

Leyman, L., De Raedt, R., Schacht, R., and Koster, E. H. (2007). Attentional biases for angry faces in unipolar depression. Psychol. Med. 37, 393-402. doi: 10.1017/S003329170600910X

Li, W., Zinbarg, R. E., and Paller, K. A. (2007). Trait anxiety modulates supraliminal and subliminal threat: brain potential evidence for early and late processing influences. Cogn. Affect. Behav. Neurosci. 7, 25-36. doi: 10.3758/CABN.7.1.25

Luck, S. J., Woodman, G. F., and Vogel, E. K. (2000). Event-related potential studies of attention. Trends Cogn. Sci. 4, 432-440. doi: 10.1016/S1364-6613(00)01545-X 
Markela-Larenc, J., Kaiser, S., Golz, T., Fiedler, P., Mundt, C., and Weisbrod, M. (2011). Attentional bias in depressive patients and the moderating effect of concurrent anxiety. Psychopathology 44, 193-200. doi: 10.1159/000319370

Martin, M., Williams, R. M., and Clark, D. M. (1991). Does anxiety lead to selective processing of threat-related information? Behav. Res. Ther. 29, 147-160. doi: 10.1016/0005-7967(91)90043-3

McCabe, S. B., and Gotlib, I. H. (1995). Selective attention and clinical depression: performance on a deployment-of-attention task. J. Abnorm. Psychol. 104, 241245. doi: 10.1037/0021-843X.104.1.241

McCabe, S. B., Gotlib, I. H., and Martin, R. A. (2000). Cognitive vulnerability for depression: deployment of attention as a function of history of depression and current mood state. Cogn. Ther. Res. 24, 427-444. doi: 10.1023/A:1005579719849

McNally, R. J. (1998). Information-processing abnormalities in anxiety disorders: implications for cognitive neuroscience. Cogn. Emot. 12, 479-495. doi: $10.1080 / 026999398379682$

Meyer, T. J., Miller, M. L., Metzger, R. L., and Borkovec, T. D. (1990). Development and validation of the Penn State Worry Questionnaire. Behav. Res. Ther. 28 487-495. doi: 10.1016/0005-7967(90)90135-6

Mogg, K., and Bradley, B. P. (2005). Attentional bias in generalized anxiety disorder versus depressive disorder. Cogn. Ther. Res. 29, 29-45. doi: 10.1007/s10608-0051646-y

Mogg, K., Bradley, B. P., Miles, F., and Dixon, R. (2004). Time course of attentional bias for threat scenes: testing the vigilance-avoidance hypothesis. Cogn. Emot. 18, 689-700. doi: 10.1080/02699930341000158

Molina, S., and Borkovec, T. D. (1994). “The Penn State Worry Questionnaire: psychometric properties and associated characteristics," in Worrying: Perspective on Theory, Assessment, and Treatment, eds G. C. L. Davey and F. Tallis (Chichester: Wiley), 265-283.

Nitschke, J. B., Heller, W., Imig, J. C., McDonald, R. P., and Miller, G. A. (2001) Distinguishing dimensions of anxiety and depression. Cogn. Ther. Res. 25, 1-22. doi: 10.1023/A:1026485530405

Nitschke, J. B., Miller, G. A., and Cook, E. W. (1998). Digital filtering in EEG/ERP analysis: some technical and empirical comparisons. Behav. Res. Methods Instrum. Comput. 30, 54-67. doi: 10.3758/BF03209416

Nolen-Hoeksema, S. (2001). Gender differences in depression. Curr. Dir. Psychol. Sci. 10, 173-176. doi: 10.1111/1467-8721.00142

Nunez, P. L., Silberstein, R. B., Shi, Z., Carpenter, M. R., Srinivasan, R., Tucker, D. M. et al. (1999). EEG coherency II: experimental comparisons of multiple measures. Clin. Neurophysiol. 110, 469-486. doi: 10.1016/S1388-2457(98)00043-1

Oldfield, R. C. (1971). The assessment and analysis of handedness: the Edinburgh Inventory. Neuropsychologia 9, 97-113. doi: 10.1016/0028-3932(71)90067-4

Perrin, F., Pernier, J., Bertrand, O., and Echallier, J. F. (1989). Spherical splines for scalp potential and current density mapping. Electroencephalogr. Clin. Neurophysiol. 72, 184-187. doi: 10.1016/0013-4694(89)90180-6

Rossignol, M., Phillippot, P., Crommelinck, M., and Campanella, S. (2008). Visua processing of emotional expressions in mixed anxious-depressed subclinical state: an event-related potential study on a female sample. Clin. Neurophysiol. 38, 267275. doi: 10.1016/j.neucli.2008.07.007

Ruz, M., and Nobre, A. C. (2008). Attention modulates initial stages of visual word processing. J. Cogn. Neurosci. 20, 1727-1736. doi: 10.1162/jocn.2008.20119

Sass, S. M., Heller, W., Stewart, J. L., Silton, R. L., Edgar, J. C., Fisher, J. E., et al. (2010). Time course of attentional bias in anxiety: emotion and gender specificity. Psychophysiology 47, 247-259. doi: 10.1111/j.1469-8986.2009.00926.x

Schupp, H. T., Junghöfer, M., Weike, A. I., and Hamm, A. O. (2004). The selective processing of briefly presented affective pictures: an ERP analysis. Psychophysiology 41, 441-449. doi: 10.1111/j.1469-8986.2004.00174.x

Stewart, J. L., Silton, R. L., Sass, S. M., Fisher, J. E., Edgar, J. C., Heller, W., et al (2010). Attentional bias to negative emotion as a function of approach and withdrawal anger styles: an ERP investigation. Int. J. Psychophysiol. 76, 9-18. doi: 10.1016/j.ijpsycho.2010.01.008

Stewart, J. L., Towers, D. N., Coan, J. A., and Allen, J. J. (2011). The oftneglected role of parietal EEG asymmetry and risk for major depressive disorder. Psychophysiology 48, 82-95. doi: 10.1111/j.1469-8986.2010.01035.x

Sumich, A. L., Kumari, V., Heasman, B. C., Gordon, E., and Brammer, M. (2006). Abnormal asymmetry of N200 and P300 event-related potentials in subclinical depression. J. Affect. Disord. 92, 171-183. doi: 10.1016/j.jad.2006.01.006
Thomas, S. J., Johnstone, S. J., and Gonsalvez, C. J. (2007). Event-related potentials during an emotional Stroop task. Int. J. Psychophysiol. 63, 221-231. doi: 10.1016/j.ijpsycho.2006.10.002

van Hooff, J. C., Dietz, K. C., Sharma, D., and Bowman, H. (2008). Neural correlates of intrusion of emotion words in a modified Stroop task. Int. J. Psychophysiol. 67, 23-34. doi: 10.1016/j.ijpsycho.2007.09.002

Watson, D. (2009). Differentiating the mood and anxiety disorders: a quadripartite model. Annu. Rev. Clin. Psychol. 5, 221-247. doi: 10.1146/annurev.clinpsy.032408.153510

Watson, D., Clark, L. A., Weber, K., Assenheimer, J. S., Strauss, M. E., and McCormick, R. A. (1995a). Testing a tripartite model: II. Exploring the symptom structure of anxiety and depression in student, adult, and patient samples. J. Abnorm. Psychol. 104, 15-25. doi: 10.1037/0021-843X. 104.1.15

Watson, D., Weber, K., Assesnheimer, J. S., Clark, L. A., Strauss, M. E., and McCormick, R. A. (1995b). Testing a tripartite model: I. Evaluating the convergent and discriminant validity of anxiety and depression symptom scales. $J$. Abnorm. Psychol. 104, 3-14. doi: 10.1037/0021-843X.104.1.3

Weber, K., Miller, G. A., Schupp, H., Borgelt, J., Awiszus, B., Popov, T., et al. (2009). Early life stress and psychiatric disorder modulate cortical responses to affective stimuli. Psychophysiology 46, 1234-1243. doi: 10.1111/j.1469-8986.2009. 00871.x

Weissman, M. M., Bland, R. C., Canino, G. J., Faravelli, C., Greenwald, S., Hwu, H.-G., et al. (1996). Cross-national epidemiology of major depression and bipolar disorder. J. Am. Med. Assoc. 276, 293-299. doi: 10.1001/jama. 276.4.293

Wells, T. T., and Beevers, C. G. (2010). Biased attention and dysphoria: manipulating selective attention reduces subsequent depressive symptoms. Cogn. Emot. 24, 719-728. doi: 10.1080/02699930802652388

West, R. (2003). Neural correlates of cognitive control and conflict detection in the Stroop and digit-location tasks. Neuropsychologia 41, 1122-1135. doi: 10.1016/S0028-3932(02)00297-X

Williams, J. M. G., Mathews, A., and MacLeod, C. (1996). The emotional Stroop task and psychopathology. Psychol. Bull. 120, 3-24. doi: 10.1037/0033-2909. 120.1 .3

Williams, L. M., Kemp, A. H., Felmingham, K., Liddell, B. J., Palmer, D. M., and Bryant, R. A. (2007). Neural biases to covert and overt signals of fear: dissociation by trait anxiety and depression. J. Cogn. Neurosci. 10, 1595-1608. doi: 10.1162/jocn.2007.19.10.1595

Wright, S. L., Langenecker, S. A., Deldin, P. J., Rapport, L. J., Nielson, K. A., Kade, A. M., et al. (2009). Gender-specific disruptions in emotion processing in younger adults with depression. Depress. Anxiety 26, 182-189. doi: 10.1002/da. 20502

Yee, C. M., and Miller, G. A. (1994). A dual-task analysis of resource allocation in dysthymia and anhedonia. J. Abnorm. Psychol. 103, 625-636. doi: 10.1037/0021843X.103.4.625

Conflict of Interest Statement: The authors declare that the research was conducted in the absence of any commercial or financial relationships that could be construed as a potential conflict of interest.

Received: 09 December 2013; accepted: 23 March 2014; published online: 09 April 2014.

Citation: Sass SM, Heller W, Fisher JE, Silton RL, Stewart JL, Crocker LD, Edgar JC, Mimnaugh KJ and Miller GA (2014) Electrophysiological evidence of the time course of attentional bias in non-patients reporting symptoms of depression with and without co-occurring anxiety. Front. Psychol. 5:301. doi: 10.3389/fpsyg.2014.00301

This article was submitted to Personality Science and Individual Differences, a section of the journal Frontiers in Psychology.

Copyright (C) 2014 Sass, Heller, Fisher, Silton, Stewart, Crocker, Edgar, Mimnaugh and Miller. This is an open-access article distributed under the terms of the Creative Commons Attribution License (CC BY). The use, distribution or reproduction in other forums is permitted, provided the original author(s) or licensor are credited and that the original publication in this journal is cited, in accordance with accepted academic practice. No use, distribution or reproduction is permitted which does not comply with these terms. 